\title{
Gamma Ray Multiplicity of 252Cf Spontaneous Fission using LiBerACE
}

D. L. Bleuel, L. A. Bernstein, J. T. Burke, M. D. Heffner, E. B. Norman, N. D. Scielzo, S. A. Sheets, N. J. Snyderman, J. Gibelin, L. W. Phair, M. Wiedeking, J. Mintz

January 18, 2008

Fourth International Conference on Fission and Properties of Neutron-Rich Nuclei Sanibel Island, FL, United States November 11, 2007 through November 17, 2007 
This document was prepared as an account of work sponsored by an agency of the United States government. Neither the United States government nor Lawrence Livermore National Security, LLC, nor any of their employees makes any warranty, expressed or implied, or assumes any legal liability or responsibility for the accuracy, completeness, or usefulness of any information, apparatus, product, or process disclosed, or represents that its use would not infringe privately owned rights. Reference herein to any specific commercial product, process, or service by trade name, trademark, manufacturer, or otherwise does not necessarily constitute or imply its endorsement, recommendation, or favoring by the United States government or Lawrence Livermore National Security, LLC. The views and opinions of authors expressed herein do not necessarily state or reflect those of the United States government or Lawrence Livermore National Security, LLC, and shall not be used for advertising or product endorsement purposes. 


\title{
GAMMA RAY MULTIPLICITY OF ${ }^{252}$ CF SPONTANEOUS FISSION USING LIBERACE*
}

\author{
D.L. BLEUEL, L.A. BERNSTEIN, J.T. BURKE, M.D. HEFFNER, E.B. NORMAN, \\ N.D. SCIELZO, S.A. SHEETS, N.J. SNYDERMAN \\ Lawrence Livermore National Laboratory, Livermore, CA 94550, USA
}

J. GIBELIN, L.W. PHAIR, M. WIEDEKING

Lawrence Berkeley National Laboratory, Berkeley, CA 94720, USA

\section{J. MINTZ}

Nuclear Engineering Department, University of California, Berkeley, CA 94720, USA

\begin{abstract}
We are studying the gamma ray and neutron multiplicity of various fission processes, beginning with the spontaneous fission of ${ }^{252} \mathrm{Cf}$, for a variety of basic and applied science purposes. The Livermore-Berkeley Array for Collaborative Experiments (LiBerACE) consists of six high-purity germanium Clover detectors (HPGe) each enclosed by an array of 16 bismuth-germanate (BGO) detectors. These detectors were arranged in a cubic pattern around a $1 \mu \mathrm{Ci}^{252} \mathrm{Cf}$ source to attempt to cover as much solid angle of gamma ray emission as possible with a high level of segmentation. The single-gamma detector response function is determined at several energies by tagging in a HPGe detector on the photopeak of one of two gamma rays in two-gamma ray calibration sources and observing the multiplicity of the remainder of the array. Summing these single-gamma responses in groups yields the response function of the array to higher multiplicity events, which are convolved with multiplicity distributions from theoretical models and compared to the measured results to test the models' validity.
\end{abstract}

\section{Introduction}

The precise probability distribution of gamma ray multiplicity is important to a variety of nuclear applications, especially passive assays of fissile materials. For instance, a multiplying medium of fissile material may be detectable inside cargo by the characteristic time signature of prompt fission gamma rays in

\footnotetext{
* This work was performed under the auspices of the U.S. Department of Energy by Lawrence Livermore National Laboratory in part under Contract W-7405-Eng-48 and in part under Contract DE-AC52-07NA27344. Part of this work was performed under the auspices of the U.S. Department of Energy by Lawrence Berkeley National Laboratory under Contract No. DE-AC0205CH11231. LLNL-CONF-400574
} 
several-generation chain reactions. The ability to detect materials depends highly on the probability of high multiplicity events.

There has been surprisingly little published work of the detailed multiplicity probability distributions of fissioning systems. Most work has reported only general properties, such as the average energy dissipated, the average multiplicity, or the peak multiplicity [1-4]. Brunson [5] attempted to determine the multiplicity distribution of ${ }^{252} \mathrm{Cf}$ in an experiment at Los Alamos National Laboratory (LANL). However, that study suffered from a low degree of segmentation, fitting a double Poisson extending beyond multiplicities of 20 to data from only eight detectors. Recent theoretical Monte Carlo simulations [6], also at LANL, have predicted good, but not exact agreement with this experiment, especially at high multiplicities, shown in Figure 1.

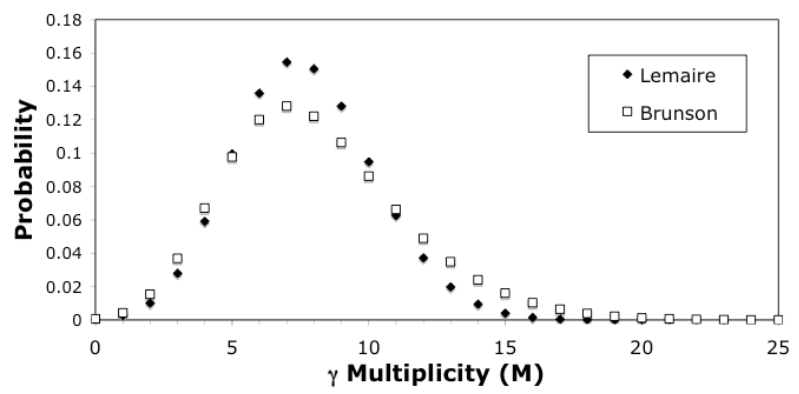

Figure 1: Bruson [5] and Lemaire [6] gamma ray multiplicity distribution for ${ }^{252} \mathrm{Cf}$ spontaneous fission.

While we are primarily interested in neutron-induced fission of uranium and plutonium, confirming the validity of the LANL theoretical model with an easily-tested ${ }^{252} \mathrm{Cf}$ source gives confidence in using it to predict more applicable neutron-induced multiplicity distributions.

\section{Experiment}

The experiment was performed using the six high-purity germanium Clover detectors (HPGe) that comprise the Livermore-Berkeley Array for Collaborative Experiments (LiBerACE) at the 88-Inch Cyclotron at Lawrence Berkeley National Laboratory (LBNL) in March of 2007. These detectors are each segmented into four crystals for a total of 24 independent detectors. Each Clover was surrounded by an array of 16 bismuth-germanate (BGO) detectors, normally used as Compton suppression shields. These six HPGe/BGO detector modules were mounted along orthogonal axes with their front faces edge-toedge in a cubic pattern in order to maximize the solid angle exposed to a source located at the center of the array. This detector arrangement provided a high 
degree of segmentation (potentially 24 HPGe and 96 BGO detectors, though 5 HPGe and 2 BGO detectors were not operational) while also providing the ability to identify specific isotopes due to the high resolution of HPGe. The four table-mounted horizontal-plane detectors were surrounded on three sides (top, left and right) by $25 \%$ density lead bricks to help minimize room background. The single-photon detection efficiency (for a $662 \mathrm{keV}$ gamma ray) of this array, measured with a calibrated ${ }^{137} \mathrm{Cs}$ source, was $55 \%$.

We then placed a $1 \mu \mathrm{Ci}^{252} \mathrm{Cf}$ liquid $(1 \mathrm{ml})$ source at the center of this array to measure the gamma multiplicity from spontaneous fission and counted for about one week. We also counted room background for about two days as well as five calibrations sources $\left({ }^{152} \mathrm{Eu},{ }^{60} \mathrm{Co},{ }^{137} \mathrm{Cs},{ }^{228} \mathrm{Th},{ }^{54} \mathrm{Mn}\right)$.

The acquisition system was set to trigger on a single photon event and record all coincident photons detected within a $4 \mu$ s time window. More precise timing information was recorded, but not used, as about $10 \%$ of the data lacked such information for unknown reasons and we did not wish to introduce bias. A $1 \mu \mathrm{Ci}{ }^{252} \mathrm{Cf}$ source, with a $3.1 \%$ spontaneous fission branching ratio, will undergo 1150 fissions per second. The number of random, uncorrelated fissions in a source of this strength occurring within a $4 \mu$ s time window is $0.46 \%$. However, most of the gamma rays emitted from a ${ }^{252} \mathrm{Cf}$ source are from the betadecay of fission products, known as "beta-delayed gammas." Our detector array was also sensitive to background radiation. The count rate with the ${ }^{252} \mathrm{Cf}$ source at the center of the array was about $6500 \mathrm{~Hz}$, about $2800 \mathrm{~Hz}$ of which was from background sources (measurable when the source was removed). At this rate, within a $4 \mu$ s timing window the number of detected events that are uncorrelated with the triggering gamma ("randoms") is about $2.6 \%$.

\section{Analysis}

Two factors primarily affect the difference between the actual source multiplicity, $\mathrm{M}$, and the detected source multiplicity, $\mathrm{M}^{\prime}$ : detector array efficiency and Compton scattering. While lower detector array efficiency lowers $\mathrm{M}^{\prime}$ relative to $\mathrm{M}$, Compton scatters in which energy from a single photon is deposited in two or more detectors raise $\mathrm{M}^{\prime}$. Rather than attempt to unfold our detected $\mathrm{M}^{\prime}$ distribution to determine a source $\mathrm{M}$ distribution, we compared our results with various experimental and theoretical $\mathrm{M}$ distributions by convolving them through the response function of our array.

To determine this response function at various source photon energies, we measured calibrated sources with known two-photon-only decays, such as Co-60, which emits exactly two photons of $1173 \mathrm{keV}$ and $1333 \mathrm{keV}$ in $99.9 \%$ of beta decays. When the full energy of one of these photons is detected in a HPGe detector, the response of the remainder of the array to a single photon of the 
other energy is determined. Summing these events in groups of M determines the M-photon response function at these specific energies. The response function to four energies ( $344 \mathrm{keV}, 1173 \mathrm{keV}, 1333 \mathrm{keV}$, and $2615 \mathrm{keV})$ was determined from two-photon-only decay branches in three calibration sources $\left({ }^{152} \mathrm{Eu},{ }^{60} \mathrm{Co}\right.$, and $\left.{ }^{228} \mathrm{Th}\right)$ and plotted for two values of $\mathrm{M}(\mathrm{M}=1,10)$ in Figure 2. The response function appears to differ only slightly as a function of photon energy, with the most notable difference at the lowest energy of $344 \mathrm{keV}$. This difference lessens as the source multiplicity increases.
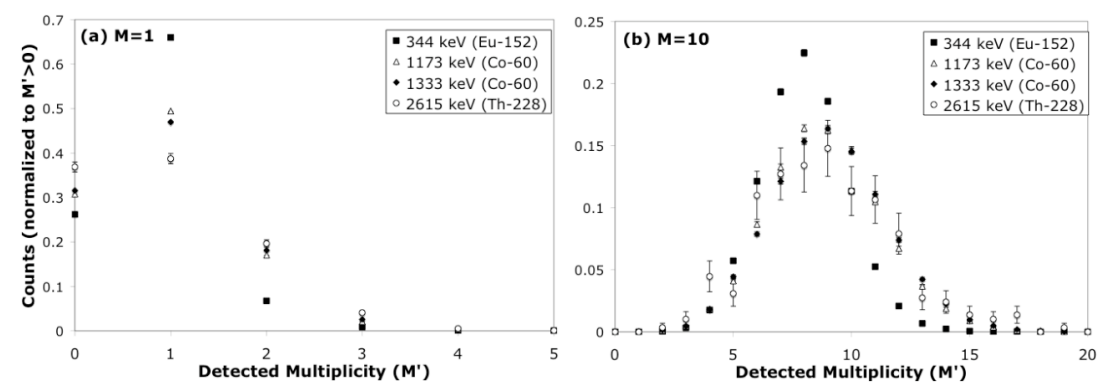

Figure 2: The detected multiplicity ( $\mathrm{M}^{\prime}$ ) response function for a source multiplicity of (a) $M=1$ and (b) $M=10$. The functions are normalized such that the sum of counts with $M^{\prime}>0$ is equal to unity.

Figure 3 shows the gamma ray spectrum seen by the HPGe detectors, divided into four regions above a $100 \mathrm{keV}$ threshold. The borders of these regions correspond to the midpoints between the four energies at which the response functions were calculated.

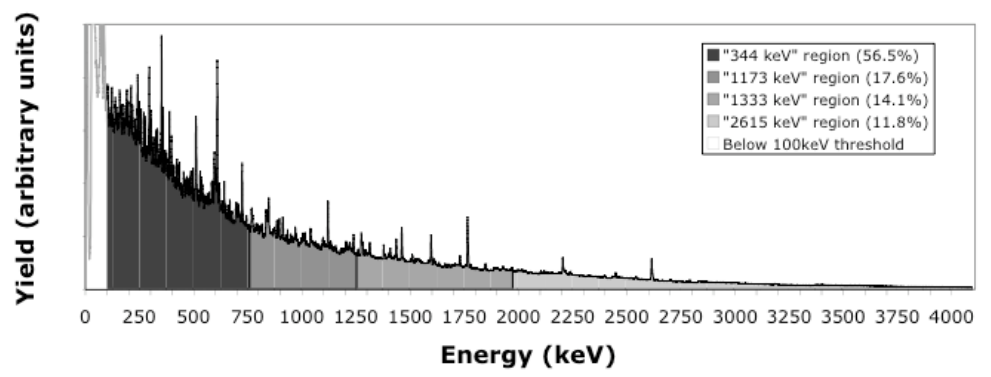

Figure 3: Efficiency-corrected HPGe spectrum.

An appropriate energy-dependent response function for a ${ }^{252} \mathrm{Cf}$ fission source was estimated by summing the four single-energy response functions for each source multiplicity, weighted by the fraction of the spectrum detected in these four regions. It should be noted that this method overestimates the contribution of lower energies, as the detected source spectrum includes some incomplete photon energy deposition from Compton scatter. 


\section{Results}

The detected multiplicity spectrum $\left(\mathrm{M}^{\prime}\right)$ in Figure 4 shows four main sources of gamma rays: room background, cosmic ray background, fission product beta decays, and fission events.

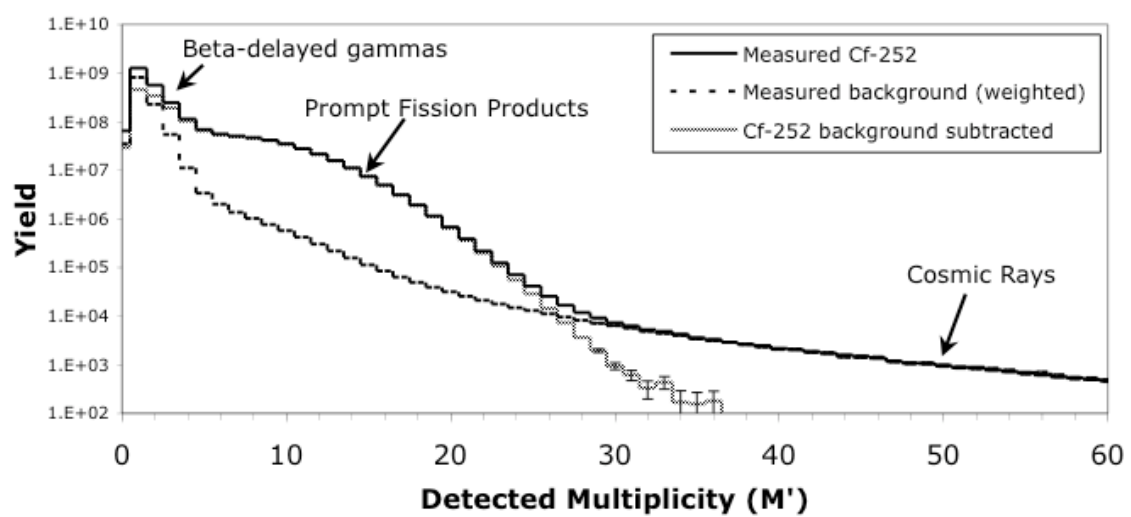

Figure 4: Measured multiplicity spectra on a logarithmic scale to show all features. Error bars were omitted when smaller than the data line. The background data was weighted by the ratio of ${ }^{252} \mathrm{Cf}$ to background data acquisition times.

Both background sources are easily subtracted by counting with no source present. The overlap of these spectra in the very high multiplicity region $\left(\mathrm{M}^{\prime}>\sim 30\right)$ of the source spectrum, dominated by cosmic ray muon interactions, gives high confidence to this background subtraction.

Because of the lack of a fission detector in this experiment, most of the photons detected were from the beta decay of fission products. These are overwhelmingly low-multiplicity events, with $\mathrm{M} \leq \sim 3-4$. These events unfortunately cannot be separated from the prompt fission multiplicity spectrum except conceptually. However, we have reasonable confidence that the probability of detecting $\mathrm{M}^{\prime}>7$ from a fission product beta decay is negligible, and we can restrict our comparisons to this region.

To determine the detected multiplicity spectra $\left(\mathrm{M}^{\prime}\right)$ that the LANL experiment [5] and theory [6] would produce in our array, the multiplicity spectra (M) from Figure 1 were convolved with the spectrum-weighted response functions described in Section 3. Figure 5 compares these with our measured $\mathrm{M}^{\prime}$ spectrum. Because the HPGe source from Figure 3 is low-energy weighted compared to the actual fission spectrum, we also compared our measured results with the LANL experiment and theory convolved with the array response function at $1333 \mathrm{keV}$. This is nearly identical to the responses at both $1173 \mathrm{keV}$ and $2615 \mathrm{keV}$ and overestimates the multiplicity spectrum as an upper bound. 

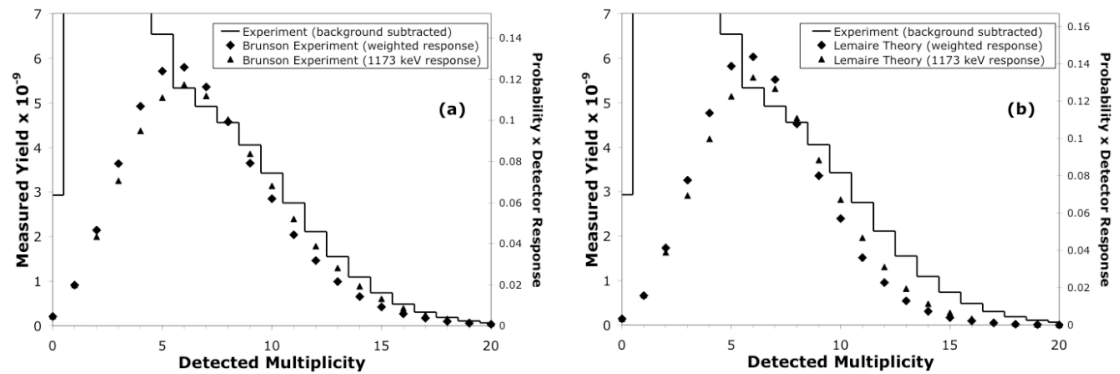

Figure 5: Comparison of our measured multiplicity spectrum (left x-axis) with (a) the LANL experiment [5] and (b) the LANL theory [6] as they would be seen in our detector array (right xaxis) using two different response functions. Scales have been roughly normalized to the datapoint at $\mathrm{M}^{\prime}=8$, where the contribution from beta-delayed gammas is expected to be negligible.

While the beta-delayed gamma spectrum makes it difficult to quantify, it appears the centroid of our measured spectrum is similar to those being compared. However, in the $\mathrm{M}^{\prime}>7$ region, both the LANL experiment and theory drop off more rapidly with higher multiplicity than our measurement, reaching zero yield at $\mathrm{M}^{\prime}=20$. This difference appears slight when comparing to a higherenergy response function of the LANL experiment, though the centroid at $\mathrm{M}^{\prime}=6$ should be lower than we measured due to beta-delayed gamma contamination.

This suggests that the actual multiplicity spectrum (M) from ${ }^{252} \mathrm{Cf}$ fission may be somewhat broader than previously measured and significantly broader than that predicted by theory. However, we are restricted to comparisons in the "uncontaminated" region of high multiplicity. A similar experiment with a fission product detector, or an alternate method of differentiating beta-delayed gammas from prompt fission gamma rays, would yield more quantitative results. Future experiments on neutron-induced fission of uranium and plutonium will employ such techniques.

Future analysis will employ tagging on gamma ray energies of known fission products in the HPGe spectrum to determine the gamma ray multiplicity dependence on neutron multiplicity.

\section{References}

1. P. Glässel, R. Schmid-Fabian, D. Schwalm, D. Habs, and H.U. v. Helmolt, Nuclear Physics, A502 315c (1989).

2. E.A. Sokol et. al., Soviet Atomic Energy 71 (5) 906-909 (1991).

3. V.S. Ramamurthy, R.K. Choudhury, J.C. Mohan, Pramana 8322 (1977).

4. R. Varma, G.K. Mehta, R.K.Choudhury, S.S. Kapoor, B.K. Nayak, V.S. Ramamurthy, Phys Rev C, 43 (4) 1850 (1991).

5. G.S. Brunson Jr., Los Alamos National Laboratory, LA-9408-T.

6. S. Lemaire, P. Talou, T. Kawano, M.B. Chadwick, and D.G. Madland, Phys $\operatorname{Rev} C$ 72, 014602 (2005). 